\title{
AUC All Normalized by Dose
}

National Cancer Institute

\section{Source}

National Cancer Institute. AUC All Normalized by Dose. NCI Thesaurus. Code C92306.

The area under the curve (AUC) from the time of dosing to the time of the last

observation divided by the dose, regardless of whether the last concentration is measurable or not. 